\title{
Research
}

\section{Out-of-hours primary care use at the end of life:}

\author{
a descriptive study
}

\begin{abstract}
Background

Out-of-hours $(\mathrm{OOH})$ primary care services are integral to the care of patients at end of life.

Little is known about the $\mathrm{OOH}$ service usage of patients with palliative care needs.

\section{Aim}

To describe patterns of usage of patients presenting to an $\mathrm{OOH}$ service and coded as palliative'
\end{abstract}

\section{Design and setting}

A descriptive study of data from the Oxfordshire $\mathrm{OOH}$ service.

\section{Method}

A database of all patient contacts with the Oxfordshire $\mathrm{OOH}$ service from a 4-year period (June 2010-August 2014) was used to extract demographic and service usage data for all contacts to which clinicians had applied a 'palliative' code. Observed differences in demographic features between palliative and non-palliative contacts were tested using logistic regression.

\section{Results}

Out of a total of 496931 contacts, there were 6045 contacts coded palliative; those 'palliative' contacts provided care to 3760 patients. Patients contacting the $\mathrm{OOH}$ service with palliative care needs did so predominantly during weekend daytime periods, and over a third had more than one contact. Patients were predictably older than the average population, but contacts coded as 'palliative' were relatively less deprived than contacts to the $\mathrm{OOH}$ service for all causes, even after adjusting for age and sex.

\section{Conclusion}

The current 'one-size-fits-most' model of $\mathrm{OOH}$ primary care may not allow for the specific needs of patients at the end of life. Wider analysis of palliative patient flow through urgent care services is needed to identify whether healthcare access at the end of life is inequitable, as well as the capacity requirements of a community-based service that can provide high-quality end-of-life care.

\section{Keywords}

ambulatory care; out-of-hours care; palliative care; service design

\section{INTRODUCTION}

In recent years, the recognition that goodquality end-of-life care is a fundamental component of a modern health service has led to greater interest in and provision of palliative care services.' A focus on patient priorities has demonstrated that, although most people with terminal illness want to die at home, only a minority achieve this. ${ }^{2}$ For patients to die at home, primary care services need to provide or to coordinate the provision of end-of-life care.

In the UK, out-of-hours (OOH) GP services are an integral part of primary care provision (Appendix 1 contains a structure and case study example). In 2013-2014, $00 \mathrm{H}$ primary care in England handled around 5.8 million cases, 3.3 million of which were face-toface consultations, including 800000 home visits. ${ }^{3}$ These $\mathrm{OOH}$ GP services provide care outside of 'core' contracted hours - usually from 18:30 to 08:00, and on weekends and bank holidays. The Oxfordshire $\mathrm{OOH}$ service provides care to a population of over 600000 people, with around 120000 patient contacts per year.

Although the number of patient contacts with $\mathrm{OOH}$ primary care is small in comparison with in-hours primary care (in which an estimated 340 million consultations occur each year), $4 \mathrm{OOH}$ services provide clinical cover for over twothirds of the hours in a calendar year $\mathrm{OOH}$ primary care services are necessarily integral to the care of patients at end of life.
This presents a challenge, however, and in January 2015 identifying the best ways of providing palliative care outside of working hours to avoid crises and help patients to stay in their place of choice' was identified by the Palliative and end of life care Priority Setting Partnership as the number one priority for palliative care research. ${ }^{5}$

To date, little is known about the $\mathrm{OOH}$ service usage of patients with palliative care needs, despite this information being a key first step in designing services better suited to their needs. This descriptive study analyses a large population-based dataset of patient contacts with an $\mathrm{OOH}$ service provider to better describe patterns of usage of patients presenting to the $\mathrm{OOH}$ service and labelled by the service as 'palliative'.

\section{METHOD}

In a service evaluation agreed with Oxford Health NHS Foundation Trust, a database of all patients presenting to the Oxfordshire $\mathrm{OOH}$ service over the 4 years from June 2010 to August 2014 was created from the electronic medical record used by $\mathrm{OOH}$ clinicians (SystemOne). This database did not include contacts with district nurses in the $\mathrm{OOH}$ period, as these were handled by a separate service. All patient identifiers were removed on entry to the database. Patients without an NHS number were not included, as repeat visits to the service could not be assessed. Demographic data consisted of age, sex, and Index of Multiple Deprivation
RFR Fisher, MA (Cantab), MRCP (UK), FHEA, academic clinical fellow; G Hayward, DPhil, MRCGP, MRCP, DRCOG, clinical lecturer, Nuffield Department of Primary Care Health Sciences, University of Oxford, Oxford, UK. D Lasserson, MA, MD, FRCP (Edin), MRCGP, associate professor Nuffield Department of Medicine, University of Oxford.

Address for correspondence

Rebecca FR Fisher, Nuffield Department of Primary Care Health Sciences, Radcliffe Primary
Care Building, Radcliffe Observatory Quarter. Woodstock Road, Oxford OX2 6GG, UK.

E-mail: rebecca.fisheraphc.ox.ac.uk Submitted: 7 January 2016; Editor's response: 1 March 2016; final acceptance: 22 April 2016. (c)British Journal of General Practice This is the full-length article (published online 6 Jul 2016) of an abridged version published in print. Cite this version as: Br J Gen Pract 2016; DOI: 10.3399/bjgp16X686137 


\section{How this fits in}

Identifying the best ways to provide palliative care outside of working hours has been highlighted by patients as a top priority for end-of-life care. Understanding the current service usage of patients at end of life is a key first step in designing services better suited to their needs. This study analyses 4 years of data from a large out-of-hours primary care service to describe service usage and demographics of patients using the service at end of life: who uses the service, when do they use it and what happens next?

(IMD) score. ${ }^{6}$ Service data included call volume and time period.

The time interval between assessments in the $\mathrm{OOH}$ period was calculated using calendar days that began at midnight, and timings of calls were classified as follows: 'evening', 19:00-00:00, 'overnight', 00:0108:00, 'day-time weekend', 08:01-18:59 (Saturday and Sunday).

\section{Validation of clinical coding}

At the end of each $\mathrm{OOH}$ consultation, clinicians assign at least one clinical code to the case (for example, 'cardiac'). For this study, patients at end of life were identified by searching the above database with the clinical code 'palliative' (the only end-of-life code available on 'System One'). As more than one clinical code could be assigned to each case, where 'palliative' was assigned as a secondary or tertiary code, the primary clinical code was also noted.

To validate the clinical codes applied by the $\mathrm{OOH}$ clinicians it was estimated, based on previous coding validity studies $^{7}$ that analysis of 230 records would be required
Figure 1. Age of patients presenting to the $\mathrm{OOH}$ service with a contact coded 'palliative' compared with all contacts to the $\mathrm{OOH}$ service. to establish the coding validity with a confidence level of $90 \%$ and $5 \%$ margin of error. A random selection of 230 records was obtained using SPSS, and the clinical code was compared by one author to the conclusion drawn by the clinician in the medical notes. The positive predictive value (PPV) of the clinical code for medical diagnosis or conclusion including those cases classed as miscellaneous was $90 \%$. If only those codes that positively described an established clinical presentation were assessed (for example, 'cardiac' but not 'miscellaneous' or 'referred to acute trust'), the PPV was $97.5 \%$.

\section{Analysis}

Demographic data including age and sex were obtained for patients coded as 'palliative', and were compared with all patients presenting to the $\mathrm{OOH}$ service. Deciles of IMD scores were generated from all patients contacting the Oxfordshire $\mathrm{OOH}$ service over the 4-year period, and the percentage of palliative care patient contacts in each of these deciles was compared. Where a difference in demographic features was noted in the 'palliative' group compared with the whole population presenting to $\mathrm{OOH}$, it was tested using logistic regression including the other available demographic features to explore whether variation in other demographic features could explain the observed difference. Analyses were undertaken in SPSS version 22.

\section{RESULTS}

Between June 2010 and August 2014, lexcluding 14572 contacts without an NHS number), there were 496931 contacts with the $\mathrm{OOH}$ service. Of these, 6045 contacts were coded as 'palliative' $11.2 \%$ of all contacts) to provide care for 3760 patients.

\section{Demographics}

The median age of patients deemed 'palliative' presenting to the $\mathrm{OOH}$ service was 80 years, with an interquartile range of 70-90 years (Figure 1). The median age of the overall population of patients presenting to the $\mathrm{OOH}$ service was 33 years, with an interquartile range of $15-58$ years. Of the patients with palliative care needs, $54.2 \%$ (2037) were female and $45.8 \%$ (1723) male, compared with $56.6 \%$ female and $43.4 \%$ male in the overall population presenting to $\mathrm{OOH}$.

Of the patients with 'palliative' codes, $37.8 \%$ were in the five most deprived deciles for Oxfordshire $\mathrm{OOH}$ contacts (Figure 2). To explore whether this difference was associated with the older age of patients 


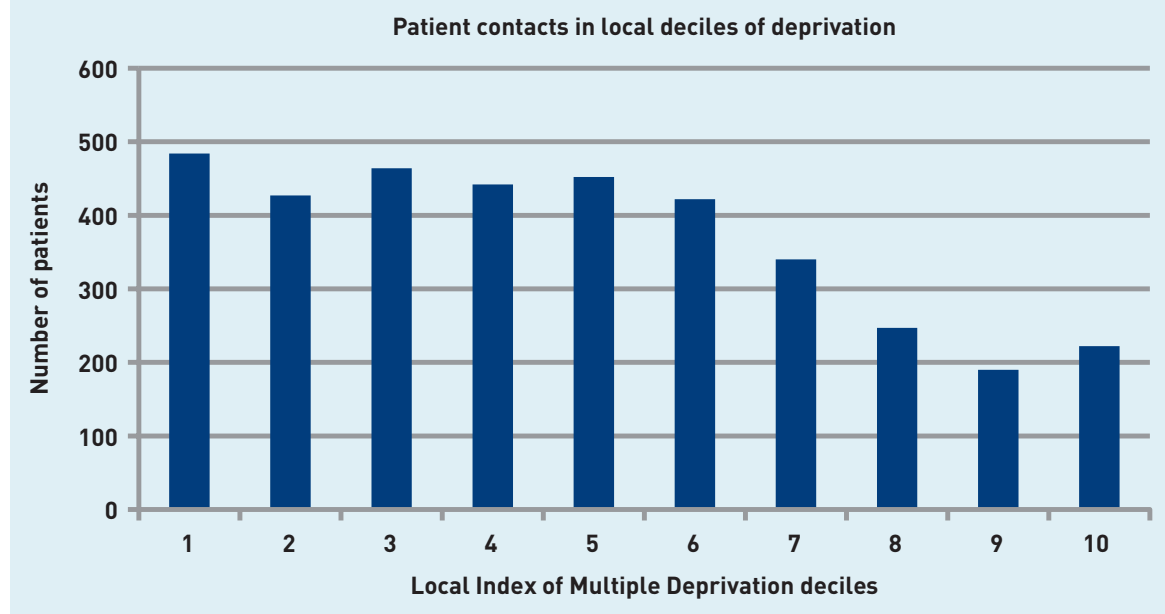

Figure 2. Number of patients coded as 'palliative' by IMD decile (local). Lower deciles are less deprived. with palliative care needs, age, sex, and IMD score were included in a multivariable logistic regression, which demonstrated a significant association between lower deprivation levels and contacting the $\mathrm{OOH}$ service with a problem coded 'palliative', in comparison with all other patient contacts with the service lOR 0.991, 95\% Cl $=0.988$ to $0.994, P<0.001$ ).

\section{Patterns of service use for contacts coded 'palliative'}

Most palliative contacts (60.9\%) were made at weekends (Saturday 30.2\%, Sunday $30.7 \%$ ), with the remaining contacts spread

\section{Table 1. Disposition at end of consultation}

\begin{tabular}{llc} 
Disposition & Number of contacts & $\%$ \\
\hline Finish consultation, no follow-up & 2104 & 34.8 \\
\hline Patient to contact own GP & 1007 & 16.7 \\
\hline Own GP to follow-up & 832 & 13.8 \\
\hline Finish - GP to contact patient & 681 & 11.3 \\
\hline District nurse & 553 & 9.1 \\
\hline Other & 318 & 5.3 \\
\hline Referred to acute hospital specialty & 307 & 5.1 \\
(including emergency department) & & 2.4 \\
\hline Finish OOH follow-up & 144 & 1.3 \\
\hline Hospital at home & 81 & 0.1 \\
\hline Unable to contact patient & 3 & 0.1 \\
\hline Cancelled by patient & 5 & 0.0 \\
\hline Minor injuries unit & 2 & 0.0 \\
\hline Emergency dental service & 1 & 0.0 \\
\hline Referred to social services & 2 & 0.0 \\
\hline Did not attend appointment & 1 & 0.0 \\
\hline Directed to make routine GP appointment & 4 & 100 \\
\hline Total & 6045 & \\
\hline OoH = out of hours. & & \\
\hline
\end{tabular}

equally from Monday to Friday. Overall, $52.2 \%$ of palliative contacts were made during the daytime at weekends and public holidays, $28.5 \%$ of contacts were made during the evening, and $19.3 \%$ occurred overnight.

\section{Disposition after $\mathbf{0 O H}$ assessment}

Sixteen different outcomes resulted from palliative patient contacts (Table 1). Of the 6045 contacts, $5.1 \%$ resulted in an acute admission. Of the remaining $94.9 \%$ whose care continued in the community, $41.8 \%$ required follow-up contact with their own GP $116.7 \%$ of these cases were asked to contact their GP, while in the remainder the GP was directed to contact the patient). In terms of outcomes, $10.4 \%$ involved referral to another community-based service, such as the district nurse or hospital at home team. In $34.8 \%$ of cases no follow-up was deemed necessary at the close of the consultation. For comparison, across the whole database of $\mathrm{OOH}$ contacts (for any reason), fewer $(31.4 \%)$ contacts required follow-up with their own GP $(19.4 \%$ were asked to contact their own GP), and more $(46.6 \%)$ ended with no follow-up.

\section{Repeat contacts after initial $\mathbf{0 O H}$ assessment}

Of 3760 patients, $33.6 \%$ had at least one further contact with the service for a palliative problem. Over $6 \%$ of patients presenting to the $\mathrm{OOH}$ service with palliative care needs had four or more palliative contacts (Table 2). Where palliative patients were seen repeatedly with palliative care concerns by the $\mathrm{OOH}$ service, $64.4 \%$ of these additional contacts were within 3 days of a previous contact with the service.

\section{Primary versus secondary coding}

Of the 6045 contacts coded by clinicians as 'palliative', 'palliative care' was the primary code in 5689 cases. Where 'palliative' was not the primary code, the most common primary code applied was 'medication advice' $(n=261)$, followed by 'cardiac' ( $n=25)$, and 'repeat prescription' $(n=24)$.

\section{DISCUSSION \\ Summary}

At least $1.2 \%$ of patient contacts with the $\mathrm{OOH}$ service were for palliative problems. Given the 5.8 million contacts with $\mathrm{OOH}$ services in England per year, this would equate to around 69600 patients per year contacting $\mathrm{OOH}$ services with palliative problems. Patients contacting the $\mathrm{OOH}$ service with palliative care needs do so predominantly during weekend daytime 


\section{Table 2. Repeat presentations coded "palliative"}

\begin{tabular}{lcc} 
Number of palliative contacts & $\boldsymbol{n}$ & $\%$ \\
\hline 1 & 2498 & 66.4 \\
\hline 2 & 743 & 19.8 \\
\hline 3 & 269 & 7.2 \\
\hline 4 & 127 & 3.4 \\
\hline 5 & 65 & 1.7 \\
\hline 6 & 24 & 0.7 \\
\hline 7 & 16 & 0.4 \\
\hline 8 & 8 & 0.2 \\
\hline 9 & 5 & 0.1 \\
\hline $10-14$ & 5 & 0.1 \\
\hline Total & 3760 & 100 \\
\hline
\end{tabular}

periods, and over a third will have more than one contact with the service. Patients are predictably older than the average population, but palliative contacts were relatively less deprived than contacts to the $\mathrm{OOH}$ service for all causes, even after adjusting for age and sex.

\section{Strengths and limitations}

To the authors' knowledge, this is the first quantitative study to look at the usage of $\mathrm{OOH}$ primary care services by patients at the end of life. It adds to the qualitative exploration of the use of $\mathrm{OOH}$ services by patients at the end of life from Worth and colleagues $^{8}$ and Leydon and colleagues.? The use of electronic records by the $\mathrm{OOH}$ service has allowed a large dataset to be collected, spanning 4 years. The quality and comprehensiveness of electronic records is, however, variable. Although the clinical coding validation exercise provides some reassurance regarding the reliability of codes assigned by $\mathrm{OOH}$ service clinicians, reliance on coding practices of individual clinicians means that the number of patients coded as 'palliative' is almost certainly an under-representation of the true number of patients presenting with end-of-life problems lboth because a clinician may have chosen another code, for example, 'heart failure' to describe a patient at end of life, and also because many people at the end of life have palliative care needs that are not recognised as such). ${ }^{10}$ In addition, it is recognised that patients with palliative care needs may access services out of hours via a variety of routes lincluding a small proportion whose own GP will have opted in to provide $\mathrm{OOH}$ care), ${ }^{3}$ and this study describes only the role of the $\mathrm{OOH}$ service.

\section{Comparison with existing literature}

Although the weekend daytime period represents a fifth of the hours covered by the $\mathrm{OOH}$ service, more than half the palliative contacts with the service were made during this period. This may reflect the constant medical need of this population; symptoms such as pain and syringe driver problems being just as likely to occur at weekends. High weekend service usage by patients with end-of-life needs also coincides with overall peak usage of $\mathrm{OOH}$ services. ${ }^{3}$ Although a link between times of peak usage and longer waiting times for home visits has not been documented, this could be of importance in a population in which pain is likely to be a common presenting complaint. ${ }^{11}$

Patients contacting the $\mathrm{OOH}$ service with palliative needs were relatively less deprived than contacts for all other causes. Among other factors, the present results may reflect increased awareness of and access to $\mathrm{OOH}$ services by more affluent patients, different service usage among different demographic groups, or simply the known trend for more economically deprived communities to have poorer access to health care. ${ }^{12}$ Overall, patients in the most deprived quintile are consistently most likely to die in hospital, whereas deaths in hospices are most common in the least deprived quintile. ${ }^{13}$ Although detailed data on the underlying diagnoses of patients contacting the $\mathrm{OOH}$ service for end-of-life care needs were not available for this study, access to such data might facilitate detailed exploration of the relationship between morbidity, deprivation, and service use. As patients at the end of life can access care via multiple different routes during the $\mathrm{OOH}$ period (for example, via direct contact with a local hospice or accident and emergency), an assessment of the levels of deprivation of patients accessing services by different routes is also required to fully understand the implications of the present findings for palliative care provision.

When compared with all contacts to the $\mathrm{OOH}$ service during this period, palliative contacts were more likely to end with a recommendation for GP follow-up. Moreover, the responsibility for arranging follow-up was more likely to be placed with the GP. The increased tendency of $\mathrm{OOH}$ clinicians to hand over these patients to their GP colleagues may reflect an attempt at establishing continuity of care for a patient group likely to have ongoing medical needs, and in whom the importance of such continuity is well recognised. ${ }^{14,15}$ This would suggest that better platforms for 
information sharing specifically for patients with palliative needs could improve both quality of care and clinician job satisfaction. The evaluation of electronic tools for information sharing such as the Londonbased 'Coordinate my Care' and the Scottish 'Key Information Summary' will provide useful direction on how information is most effectively shared.

A notably small proportion of contacts coded as 'palliative' resulted in acute hospital admission (5.1\%). Although this may represent a desire on the part of clinicians to manage people at the end of life in their own homes, it is possible that more unwell patients self-triage directly to hospital services, bypassing $\mathrm{OOH}$ primary care.

\section{Implications for research and practice}

The present findings suggest that the needs of a large proportion of the patients presenting to $\mathrm{OOH}$ with palliative problems could be met by a dedicated service in the weekend daytime periods. Although some localities have linked third-sector provision of palliative care services with local hospice and hospital facilities to provide dedicated palliative care services out of hours, ${ }^{16}$ the resource implications of these services mean that for many areas of the UK the $\mathrm{OOH}$ primary care service will continue to provide the majority of unplanned endof-life care. Work is required to explore whether dedicated services could facilitate faster access and be cost-effective. The success of any such service would rely on both accurate identification of patients with palliative care needs in the community, and effective communication and information sharing between service providers.

Patients at the end of life often have different needs and time frames of need from patients without need of palliative care. Many $\mathrm{OOH}$ services are accessed in the same way whether you are a teenager with a sore throat or a patient dying in pain. It is possible that the current onesize-fits-most' model of $\mathrm{OOH}$ primary care provision does not allow for the specific needs of patients who are dying in the community. A wider analysis of palliative patient flow through $\mathrm{OOH}$ services is now needed to identify if healthcare access at the end of life is inequitable, as well as the capacity requirements of a communitybased palliative care service that can provide high-quality $24 / 7$ care for patients at the end of life.

\section{Funding}

This study did not receive any external funding. Daniel Lasserson is funded by the NIHR Oxford Biomedical Research Centre and the views expressed are those of the authors and not necessarily those of the NHS, the NIHR, or the Department of Health.

\section{Provenance}

Freely submitted; externally peer reviewed.

\section{Competing interests}

The authors have declared no competing interests.

\section{Acknowledgements}

The authors acknowledge the contribution of Dr Mary Miller, Consultant in Palliative Medicine, and Ms Helen Hunt, Clinical Lead for Urgent and Ambulatory Care Services, Oxford Health NHS Foundation Trust.

\section{Discuss this article}

Contribute and read comments about this article: bjgp.org/letters 


\section{REFERENCES}

1. Department of Health. End of life care strategy: promoting high quality care for all adults at the end of life. London: DH, 2008

2. Higginson I. Priorities and preferences for end of life care in England, Wales and Scotland. London: National Council for Hospice and Specialist Palliative Care Services, 2003.

3. National Audit Office. Out of hours GP services in England. London: National Audit Office, 2014. http://www.nao.org.uk/wp-content/uploads/2014/09/Out-ofhours-GP-services-in-England1.pdf (accessed 21 Jun 2016).

4. Deloitte. Under pressure: the funding of patient care in general practice. London: Deloitte, 2014. http://www.rcgp.org.uk/campaign-home/ /media/ Files/PPF/Deloitte\%20Report_Under\%20Pressure.ashx laccessed 21 Jun 2016).

5. Marie Curie. Palliative and end of life care Priority Setting Partnership. (PeolcPSP). 2015. https://www.mariecurie.org.uk/globalassets/media/ documents/research/PeolcPSP_Final_Report.pdf (accessed 21 Jun 2016).

6. Communities and Local Government. The English Indices of Deprivation 2010. Department for Communities and Local Government, 2011.

7. Khan NF, Harrison SE, Rose PW. Validity of diagnostic coding within the General Practice Research Database: a systematic review. Br J Gen Pract 2010; DOI: 10.3399/bjgp10X483562.

8. Worth A, Boyd K, Kendall M, et al. Out-of-hours palliative care: a qualitative study of cancer patients, carers and professionals. Br J Gen Pract 2006; 56(522): 6-13.

9. Leydon GM, Shergill NK, Campion-Smith C, et al. Discontinuity of care at end of life: a qualitative exploration of $\mathrm{OOH}$ end of life care. BMJ Support Palliat Care 2013; 3(4): 412-421.

10. Omega, the National Association for End of Life Care. End of life care in primary care: 2009 national snapshot. Shrewsbury: Omega, 2009. http:// www.goldstandardsframework.org.uk/cd-content/uploads/files/Library, $\% 20$ Tools\%20\%26\%20resources/EOLC\%20in\%20Primary\%20Care\%20national\%20 snapshot\%20-\%20Key\%20Findings.pdf (accessed 21 Jun 2016).

11. Adam R, Wassell P, Murchie P. Why do patients with cancer access out-ofhours primary care? A retrospective study. Br J Gen Pract 2014; DOI: 10.3399/ bjgp14X677158.

12. Marmot M, Allen J, Goldblatt P, et al. Fair society, healthy lives: strategic review of health inequalities in England post 2010. London: Marmot Review, 2010.

13. National End of Life Care Intelligence Network. Deprivation and death: variation in place and cause of death. Bristol: National End of Life Care Intelligence Network, 2012. http://www.endoflifecare-intelligence.org.uk/ resources/publications/deprivation_and_death (accessed 21 Jun 2016).

14. Michiels E, Deschepper R, Van Der Kelen G, et al. The role of general practitioners in continuity of care at the end of life: a qualitative study of terminally ill patients and their next of kin. Palliat Med 2007; 21(5): 409-415.

15. Burge F, Lawson B, Johnston G. Family physician continuity of care and emergency department use in end-of-life cancer care. Med Care 2003; 41(8): 992-1001.

16. Purdy S, Lasseter G, Griffin T, Wye L. Impact of the Marie Curie Cancer Care Delivering Choice Programme in Somerset and North Somerset on place of death and hospital usage: a retrospective cohort study. BMJ Support Palliat Care 2015; 5(1): 34-39. 


\section{Appendix 1. Structure of out-of-hours primary care in the UK with illustrative case study}

Out-of-hours $(\mathrm{OOH})$ primary care provides GP services outside of 'core contracted hours': from 18:30 to 08:00 Monday to Friday, and 24/7 at weekends and bank holidays. Since 2004, GPs have been able to opt out of providing 24-hour primary care cover for their practice population, and most have chosen to do so. Just $10 \%$ of practices have opted to retain responsibility for providing their own $\mathrm{OOH}$ care. ${ }^{10}$ For the remaining $90 \%$ of practices, the NHS commissions $\mathrm{OOH}$ services separately from in-hours services. Since April 2013 , NHS England has delegated responsibility for this commissioning to 211 clinical commissioning groups. Hence the actual provider of the $\mathrm{OOH}$ service, and the exact way in which that service is delivered, will vary geographically within the country.

\section{$0 \mathrm{OH}$ service in practice: a case study example from the $0 x$ fordshire $00 \mathrm{H}$ service}

Mrs Smith is 84 years old and has advanced Alzheimer's dementia. Her daughter Sarah visits after work on a Tuesday evening and is concerned about her mother. Mrs Smith is coughing, feverish, and has not eaten dinner.

Sarah phones ' 111 ' on her mother's behalf. Her call is answered by a trained call handler who works through an algorithm of questions (NHS Pathways) to determine the most appropriate and safe outcome for Sarah and her mother. The handler might call her an ambulance, direct her to the local $\mathrm{OOH}$ service, direct her to a clinician in 111, or give her simple self-management advice. In this case the handler directs Sarah to the local OOH GP service. She is told she will be contacted directly by them, and the 111 call ends. Sarah's case is now transferred to the local $\mathrm{OOH}$ provider. It appears on their screen as needing a telephone consultation, with a priority assigned by the 111 call handler. A GP, Dr Jones, working on shift at the $\mathrm{OOH}$ centre picks up the call. Dr Jones works only for the $\mathrm{OOH}$ service. Some of her colleagues have jobs in-hours' in local GP practices too and therefore may know the patient; however, most patients will be unknown to the $\mathrm{OOH} \mathrm{GP}$.

Dr Jones rings Sarah to discover more about the situation with her mother and perform a telephone clinical assessment. She does not have access to Mrs Smith's whole medical record, but, because Mrs Smith has been flagged up by her in-hours GP as near the end of life, the in-hours GP has e-mailed the OOH service some 'special notes'. These tell Dr Jones that Mrs Smith has Alzheimer's, and that she would like to be cared for at home if possible. Dr Jones listens to the history and, after assessment, decides that Mrs Smith needs to be seen for a further face-to-face assessment. Mrs Smith is unable to travel to the $\mathrm{OOH}$ centre and so Dr Jones allocates a home visit. Once this is completed (by Dr Jones, or another colleague on shift that evening), her notes will be completed and a clinical code applied to the case. This code is at the discretion of the clinician (no formal coding training is given for either in- or out-of-hours GPs). Mrs Smith's in-hours GP will immediately receive an e-mail notification from the $\mathrm{OOH}$ service containing the notes of the case so that they can see what has happened. 